\title{
The Important Anti-Apoptotic Role and Its Regulation Mechanism of PTTG1 in UV-Induced Apoptosis
}

\author{
Yongqing Lai ${ }^{1}$, Dianqi $\mathrm{Xin}^{1}$, Junhai $\mathrm{Bai}^{2}$, Zebin $\mathrm{Mao}^{2, *}$ and Yanqun $\mathrm{Na}^{1, *}$ \\ ${ }^{1}$ Department of Urology, Peking University First Hospital and Institute of Urology, Peking University, Beijing 100034, China \\ ${ }^{2}$ Department of Biochemistry and Molecular Biology, Peking University Health Science Center, Beijing 100083, China
}

Received 23 April 2007, Accepted 18 July 2007

\begin{abstract}
Pituitary tumor transforming gene (PTTG1) is widely detected in many tumors. Increasing evidence reveals that PTTG1 is associated with cell proliferation, cellular transformation and apoptosis. However, the functions of PTTG1, especially its role in DNA damage-induced apoptosis, remain largely unclear. In this report, we used UV irradiation to induce apoptosis in HeLa cells to examine the role of PTTG1 in UV-induced apoptosis by RNAi-mediated knockdown and overexpression of PTTG1. RNAi-mediated knockdown of PTTG1 expression increased and overexpression of PTTG1 decreased the UVinduced apoptosis. Furthermore, UV irradiation decreased PTTG1 mRNA and protein expression. These effects were found to be mediated by JNK pathway. Therefore, PTTG1 had an important anti-apoptotic role in UV-induced apoptosis and this role was mediated by JNK pathway. These results may provide important information for understanding the exact role and the regulation mechanism of PTTG1 in UV-induced apoptosis.
\end{abstract}

Keywords: Apoptosis, Jun N-terminal kinases, Pituitary tumor transforming gene 1, PTTG1, UV

\section{Introduction}

Pituitary tumor transforming gene (PTTG1), also called securin, is a newly isolated oncogene (Pei and Melmed, 1997; Dominguez et al., 1998). Human PTTG1 cDNA encodes a protein of 203 amino acids that is predominately located in the cytoplasm with partial nuclear localization. PTTG1 is overexpressed in various primary tumors and tumor cell lines,

* To whom correspondence should be addressed.

Tel: 86-10-82805138 (Z. M.), 86-10-66551032 (Y. N.)

Fax: 86-10-66551032 (Y. N.)

E-mail: zbmao@bjmu.edu.cn (Z. M.),nayq@bjmu.edu.cn (Y. N.) including carcinomas of lung, breast, esophagus, colon, melanoma, leukemia, lymphoma, pituitary adenoma and astrocytoma (Saez et al., 1999; Zhang et al., 1999a; Zhang et al., 1999b; Heaney et al., 2000; Shibata et al., 2002; TfeltHansen et al., 2004). However, among normal adult tissues, PTTG1 is expressed at high level only in the testes, with lower expression detected in thymus and intestines (Pei and Melmed, 1997; Dominguez et al., 1998; Zhang et al., 1999b). PTTG1 expression is reported to be a powerful marker for lymph-node invasion and tumor recurrence and a novel therapeutic target in breast cancer (Solbach et al., 2004; Ogbagabriel et al., 2005). It is also proposed to be a potential prognostic marker and therapeutic target for differentiated thyroid cancer (Boelaert et al., 2003; Saez et al., 2006).

The exact function of PTTG1 is not clear, but increasing evidence reveals that PTTG1 plays crucial roles in cell-cycle progression, appropriate cell division and chromosome stability, in addition to its involvement in malignant transformation and tumorigenesis (Zou et al., 1999; Yu et al., 2000; Jallepalli et al., 2001; Wang et al., 2001; Clem et al., 2003). PTTG1 inhibits sister chromatid separation and is related to cell-cycle control. PTTG1 expression appears to be cell-cycle dependent and peaks in mitosis. PTTG1 can be phosphorylated by Cdc2 kinase during mitosis (Ramos-Morales et al., 2000). PTTG1 processes transcriptional activation capability and directly binds to the c-myc promoter sequence and upregulates its expression, which suggests that PTTG1 would mediate cellular transformation by activating this oncogene (Pei, 2001). As an oncogene, PTTG1 is a multifunctional protein that stimulates the expression and secretion of mitogenic and angiogenic factors such as bFGF and VEGF, which implies that it may play a role in tumor angiogenesis (McCabe et al., 2003). Increased expression of PTTG1 in mouse fibroblast (NIH 3T3) and human embryonic kidney (HEK293) cells results in increased cell proliferation, induced cellular transformation and promoted tumor formation, thus demonstrating the importance of PTTG1 in human tumorigenesis (Kakar and Jennes, 1999; Hamid et al., 2005). This evidence implies that 
PTTG1 plays an important role in cell proliferation. But its role in DNA damage-induced apoptosis remains unclear.

In this report, we used UV irradiation to induce apoptosis in HeLa cells to examine the role of PTTG1 in UV-induced apoptosis by RNAi-mediated knockdown and overexpression of PTTG1. RNAi-mediated knockdown of PTTG1 expression increased and overexpression of PTTG1 decreased the UVinduced apoptosis. Furthermore, UV irradiation decreased PTTG1 mRNA and protein expression and these effects were mediated by JNK pathway.

\section{Materials and Methods}

Construction of plasmids. pIRES2-PTTG1-The human PTTG1 cDNA was generated by use of specific primers and cDNA prepared from total RNA as a template in PCR. The primers used were sense 5'-AGAATGGCTACTCTGATCTATG-3' and antisense 5'-CACAAACTCTGAAGCACTAAG-3'. The PCR conditions were initial denaturation at $95^{\circ} \mathrm{C}$ for $3 \mathrm{~min}$, then $95^{\circ} \mathrm{C}$ for $30 \mathrm{~s}, 50^{\circ} \mathrm{C}$ for $45 \mathrm{~s}$, and $72^{\circ} \mathrm{C}$ for $1 \mathrm{~min}$ for 32 cycles, with the final extension at $72^{\circ} \mathrm{C}$ for $10 \mathrm{~min}$ after the last cycle of amplification. The pIRES2PTTG1 chimeric construct was prepared by subcloning the fulllength PTTG1 cDNA into pIRES2-EGFP vector (Clontech Laboratories). The construct was confirmed by enzymatic digestion and sequenced to verify the correct reading frame.

Inducible RNAi of PTTG1-Two single-strand oligos were synthesized as follows: Top-strand oligo, 5'-CACCGTCCTCTAGA CTTTGAGAGTTTCAAGAGAACTCTCAAAGTCTAGAGGAT TTTTTGGAA-3'; and lower-strand oligo, 5'-AAAATTCCAAAAA ATCCTCTAGACTTTGAGAGTTCTCTTGAAACTCTCAAAGT CTAGAGGAC-3'. These 2 oligos were annealed to form duplexes. The duplex products were step-wise subcloned into $\mathrm{pENTR/H1/TO}$ vector (BLOCK-iT ${ }^{\mathrm{TM}}$ Inducible H1 RNAi Entry Vector Kit, Invitrogen) and confirmed by sequencing.

Cell culture, transfection and selection. The HeLa cell line was purchased from the American Type Culture Collection and cultured in Dulbecco's modified Eagle's medium (DMEM) containing 10\% fetal calf serum. All of the culture reagents and media were from Gibco. Zeocin, blasticidin, G418 and tetracycline were purchased from Invitrogen.

To establish a stable cell line that stably expressing PTTG1 and inducibly repress PTTG1, we followed the protocols of pIRES2EGFP Vector (Clontech Laboratories) and BLOCK-iT Inducible H1 RNAi Entry Vector Kit (Invitrogen), respectively. Briefly, For the establishment of the HeLa cell line stably expressing PTTG1 and vector, pIRES2-PTTG1 and pIRES2 plasmid was transfected into HeLa cellsf"respectively. After $24 \mathrm{~h}$, transfected HeLa cells were under the selection of $600 \mu \mathrm{g} / \mathrm{ml} \mathrm{G} 418$ for approximately 1 week. These two kinds of neomycin-resistant colonies were named HeLa/pIRES2 PTTG1 and HeLa/vector cells, respectively. After selection, both cell types were cultured with $10 \%$ fetal calf serum with $300 \mu \mathrm{g} / \mathrm{ml} \mathrm{G} 418$. Western blotting was performed to detect PTTG1 gene expression in these two cells.

The BLOCK-iT ${ }^{\mathrm{TM}}$ Inducible H1 RNAi Entry Vector Kit was used to generate tetracycline-regulated expression of a short hairpin
RNA (shRNA) to knockdown PTTG1 level in HeLa cells. To obtain a cell line that stably expresses the Tet repressor, pcDNA6/ TR was transfected into HeLa cells. After $24 \mathrm{~h}$, transfected cells were under the selection of $4 \mu \mathrm{g} / \mathrm{ml}$ blasticidin for approximately 1 week. The blasticidin-resistant colonies were expanded in $10-\mathrm{cm}$ dishes and cultured in media with $2 \mu \mathrm{g} / \mathrm{ml}$ blasticidin consistently expressing the same amount of Tet repressor. To generate tetracycline-regulated expression of PTTG1, pENTR/H1/TO was transfected into HeLa cells stably expressing Tet repressor. After $24 \mathrm{~h}$, transfected cells were under the selection of $50 \mu \mathrm{g} / \mathrm{ml}$ zeocin and $2 \mu \mathrm{g} / \mathrm{ml}$ blasticidin for approximately 10 days. The zeocinresistant colonies were expanded in 10-cm dishes for analysis. After selection, cells with tetracycline-regulated expression of PTTG1, named HeLa/P-T, were cultured in DMEM supplemented with $10 \%$ fetal calf serum with $25 \mu \mathrm{g} / \mathrm{ml}$ zeocin and $2 \mu \mathrm{g} / \mathrm{ml}$ blasticidin. Western blot analysis was performed to determine the alteration in PTTG1 expression on culture with or without $1 \mu \mathrm{g} / \mathrm{ml}$ tetracycline.

Western blotting. Cell lysates were prepared as reported previously (Yu et al., 2002). Briefly, cells were lysed with cell lysis buffer for $30 \mathrm{~min}$ on ice, and the lysates were clarified by centrifugation at $12,000 \times g$ for $15 \mathrm{~min}$ at $4^{\circ} \mathrm{C}$. Protein concentration was quantified (PIERCE), and protein samples $(50 \mu \mathrm{g})$ were separated by SDSPAGE and transferred onto nitrocellulose membranes. PTTG1, p$\mathrm{JNK}$, p-p38 and $\beta$-actin proteins were identified with the primary antibodies sc-22772, sc-6254, sc-7975-R and sc-47778, respectively, (Santa Cruz Biotechnology). The secondary antibodies, sc-22772 (rabbit polyclonal anti-PTTG1) and sc-7975-R (rabbit polyclonal anti-p-p38; both Santa Cruz Biotechnology) were detected with sc2004 (goat anti-rabbit IgG-HRP)£“" and sc-6254 (mouse monoclonal anti-p-JNK) and sc-47778 (mouse monoclonal anti-â-actin) were detected with sc-2005 (goat anti-mouse IgG-HRP). Each assay was repeated at least 3 times.

Apoptosis analysis by flow cytometery. Cells were challenged with UVC (UVC 500 Ultraviolet Crosslinker£aHoefer) at $200 \mathrm{~J} / \mathrm{m}^{2}$ to induce apoptosis (Nakagawa et al., 2002; Hwang et al., 2007), whereupon the monolayers were incubated in DMEM with supplements. Detached and adherent cells were then harvested at various times (0-48 h). Cells were then stained either by propidium iodide (PI) or annexin V. For PI staining, cells were analyzed for DNA content by the flow cytometry FACSCalibur (BD Biosciences). Annexin V staining involved use of ApoAlert apoptosis kits (BD Biosciences Clontech) following the manufacturer's recommendations. Annexin V-stained cells were analyzed by flow cytometry, measuring the fluorescence emission at $488 \mathrm{~nm}$. Each apoptosis assay was carried out in triplicate and repeated at least 3 times.

Semi-quantitative real-time PCR. Total RNA was extracted with use of RNeasy kit (Qiagen). cDNA was prepared from $2 \mu \mathrm{g}$ of total RNA (TaqMan Reverse Transcription Reagents, ABI/Roche, Branchburg, NJ) following the manufacturer's instructions. PCR amplifications involved the SYBR Green PCR Master Mix kit (Applied Biosystems) according to the manufacturer's protocol in a real-time ABI PRISM 7700 Instrument (PE Applied Biosystems). We used the standard curve method to quantify and normalize amounts of PTTG1 relative to GAPDH mRNAs. Two pairs of primers were synthesized as follows: PTTG1 primer, forward 5'- 
TGA TCC TTG ACG AGG AGA GAG-3', reverse 5'-GGT GGC AAT TCA ACA TCC AGG-3'; GAPDH primer, forward 5'-CAC CAG GGC TGC TTT TAA CTC-3', reverse 5'-GAA GAT GGT GAT GGG ATT TC-3'. Each assay was repeated at least 3 times.

\section{Results}

Suppression of PTTG1 increases UV-dependent apoptosis. As shown in Fig. 1A, the expression of PTTG1 protein decreased markedly in HeLa/P-T cells on incubation with 1 $\mu \mathrm{g} / \mathrm{ml}$ tetracycline for $24 \mathrm{~h}$.

To elucidate the role of PTTG1 in UV-induced apoptosis, we performed flow cytometric analysis to determine the rate of UV-dependent cell death in normal HeLa cells and HeLa/P$\mathrm{T}$ cells. Both of these 2 cell types were split into 2 subpopulations: one incubated in normal media and the other in media with $1 \mu \mathrm{g} / \mathrm{ml}$ tetracycline for $24 \mathrm{~h}$ before challenge with UV-C fluence of $200 \mathrm{~J} / \mathrm{m}^{2}$. Irradiated cells were reincubated in their corresponding media for different times $(0$ $48 \mathrm{~h}$ ). Only a minor fraction (about $1 \sim 3 \%$ ) of the nonirradiated cells underwent apoptosis. As shown in Fig. 1B, the proportion of UV-induced apoptosis in $\mathrm{HeLa} / \mathrm{P}-\mathrm{T}$ cells incubated with $1 \mu \mathrm{g} / \mathrm{ml}$ tetracycline was markedly larger than that of other 3 subpopulations (no apparent difference between these 3 subpopulations).

The role of PTTG1 in UV-induced apoptosis was also analyzed by fluorescent annexin $\mathrm{V}$ staining. Both of those 2 cell types were treated as described above and stained with annexin V. Fig. 1C showed that UV-induced apoptosis was greater in $\mathrm{HeLa} / \mathrm{P}-\mathrm{T}$ cells incubated with tetracycline than in the other 3 subpopulations. At $24 \mathrm{~h}$ after UV treatment, $48 \%$ of HeLa/P-T cells with tetracycline underwent apoptosis as compared with about $33 \%$ of other 3 subpopulations (no apparent difference between these 3 subpopulations). Together, these results suggest that suppression of PTTG1 increased UV-induced apoptosis.

Overexpression of PTTG1 decreases UV-dependent apoptosis. Since the knockdown of PTTG1 increased UVinduced apoptosis, we reasoned that PTTG1 protected the HeLa cell line from UV-induced apoptosis. Therefore, we determined the role of PTTG1 in UV-induced apoptosis by western blotting to detect PTTG1 protein expression in HeLa/ pIRES2 PTTG1 and HeLa/vector cell types. The expression of PTTG1 protein in HeLa/pIRES2 PTTG1 cells was increased markedly as compared with that of the other 2 groups (Fig. 2A).

We also performed flow cytometric analysis to determine the rate of UV-induced cell death in normal HeLa, HeLa/ pIRES2 PTTG1 and HeLa/vector cells challenged with UV-C fluence of $200 \mathrm{~J} / \mathrm{m}^{2}$. Irradiated cells were re-incubated in media for different times (0-48 h). Only a minor fraction (about 1 3\%) of the nonirradiated cells underwent apoptosis. As shown in Fig. 2B, the proportion of UV-induced apoptosis in HeLa/pIRES2 PTTG1 cell line was markedly smaller than
(A)
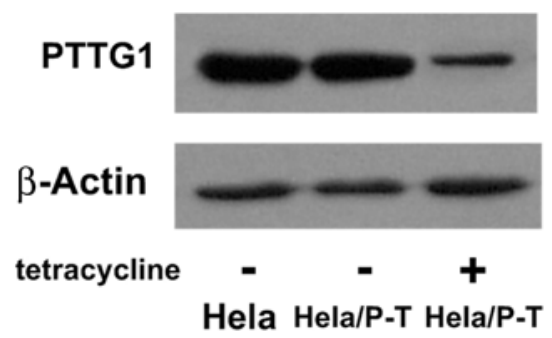

(B)
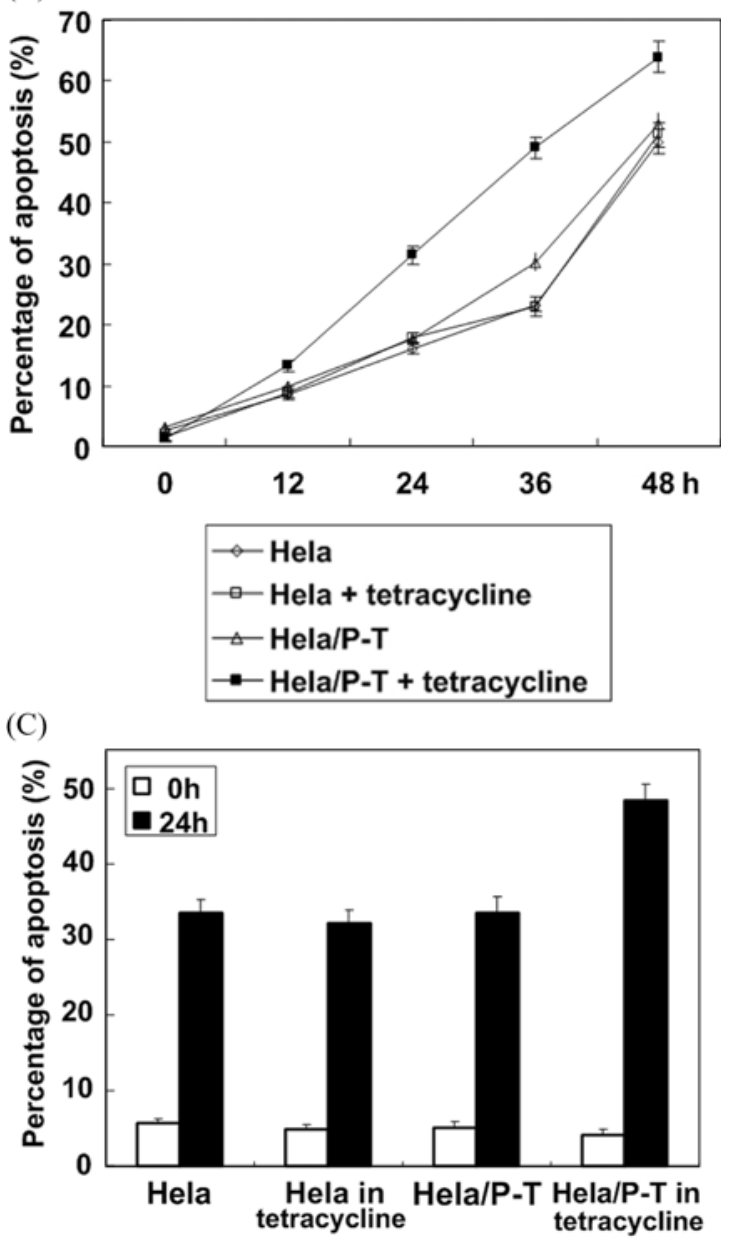

Fig. 1. Expression of PTTG1 and UV-dependent apoptotic response of cell lines HeLa, HeLa in tetracycline, HeLa/P-T and HeLa/P$\mathrm{T}$ in tetracycline. (A) Expression of PTTG1 in cell lines HeLa, HeLa/P-T and HeLa/P-T in $1 \mu \mathrm{g} / \mathrm{ml}$ tetracycline. The first 2 cell types were incubated in normal DMEM media and the last one in DMEM with $1 \mu \mathrm{g} / \mathrm{ml}$ tetracycline for $24 \mathrm{~h}$ before harvesting. (B-C) UV-dependent apoptotic response of cell lines HeLa, HeLa in tetracycline, HeLa/P-T and HeLa/P-T in tetracycline. Two cell lines (HeLa and HeLa/P-T) were divided into 2 subpopulations, one incubated in normal DMEM, the other in DMEM with 1 $\mu \mathrm{g} / \mathrm{ml}$ tetracycline, then incubated in corresponding media for $24 \mathrm{~h}$ before UV irradiation $\left(200 \mathrm{~J} / \mathrm{m}^{2}\right)$ and reincubated in their corresponding media. Apoptotic fractions were determined by flow cytometry at the indicated times. (B) The percentage of apoptotic cells following UV irradiation then harvested at 0,12 , 24,36 and $48 \mathrm{~h}$ and analyzed with flow cytometry. (C) The percentage of apoptotic cells following UV irradiation then harvested at either immediately $(0 \mathrm{~h})$ or $24 \mathrm{~h}$ later, then Annexin $\mathrm{V}$ stained, and analyzed with flow cytometry. Data are presented as means \pm SEM of three independent experiments. 
(A)
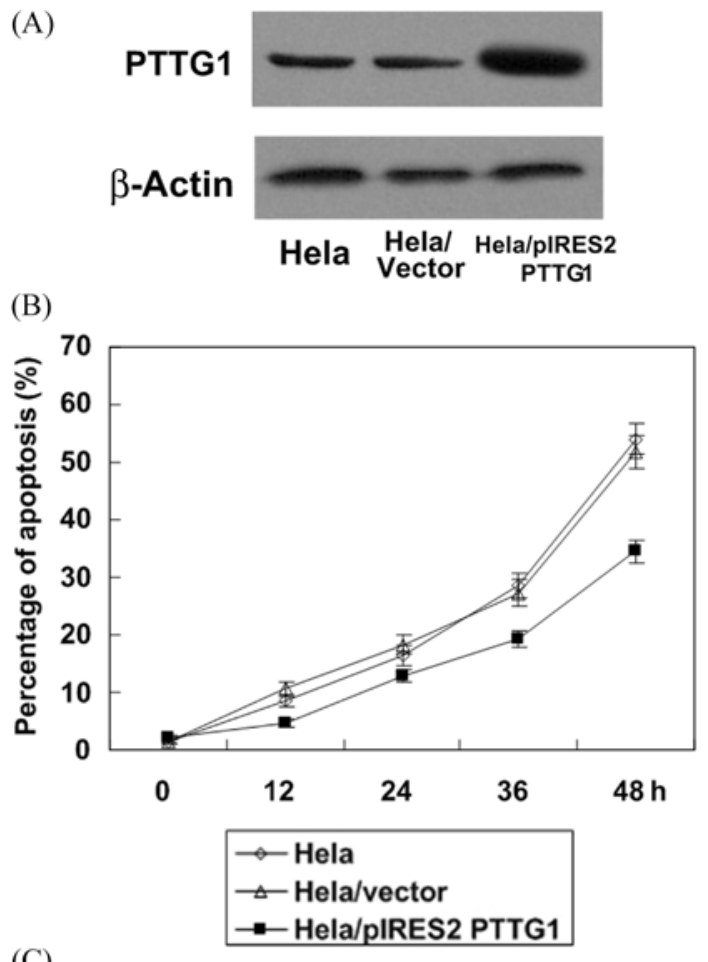

(C)

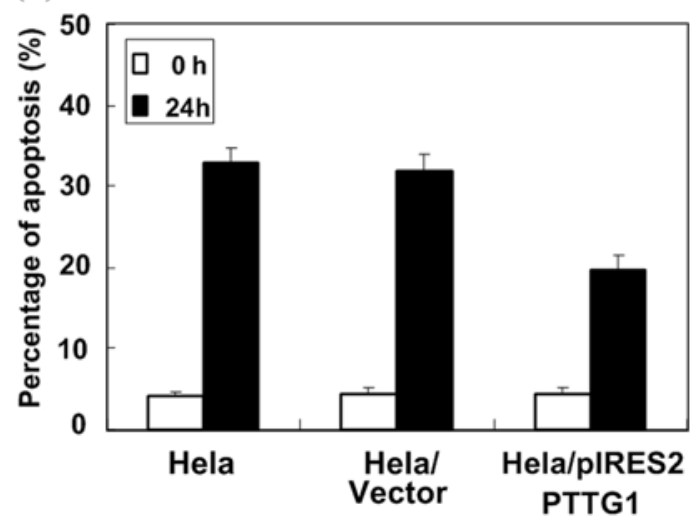

Fig. 2. Expression of PTTG1 and UV-dependent apoptotic response of cell lines HeLa, HeLa/Vector, HeLa/pIRES2 PTTG1. (A) Expression of PTTG1 in cell lines Hela, Hela/Vecror and Hela/ pIRES2 PTTG1. All the 3 cell lines were incubated in normal DMEM media. (B-C) UV-dependent apoptotic response of cell lines HeLa, HeLa/Vector, HeLa/pIRES2 PTTG1. Cell lines were UV challenged $\left(200 \mathrm{~J} / \mathrm{m}^{2}\right)$ and reincubated in DMEM. Apoptotic fractions were determined by flow cytometry at the indicated times. (C) The percentage of apoptotic cells following UV irradiation then harvested at either immediately $(0 \mathrm{~h})$ or $24 \mathrm{~h}$ later, then Annexin V stained, and analyzed with flow cytometry. Data are presented as means $\pm \mathrm{SEM}$ of three independent experiments.

that of normal HeLa cells and HeLa/vector cells (no apparent difference between these 2 groups).

The role of PTTG1 in UV-induced apoptosis was also analyzed by fluorescent annexin V staining. Fig. 2C showed that UV-induced apoptosis was less in HeLa/pIRES2 PTTG1 cells than in the other 2 cells. At $24 \mathrm{~h}$ after UV treatment, about $32 \%$ of HeLa/pIRES2 PTTG1 cells underwent apoptosis,
(A)
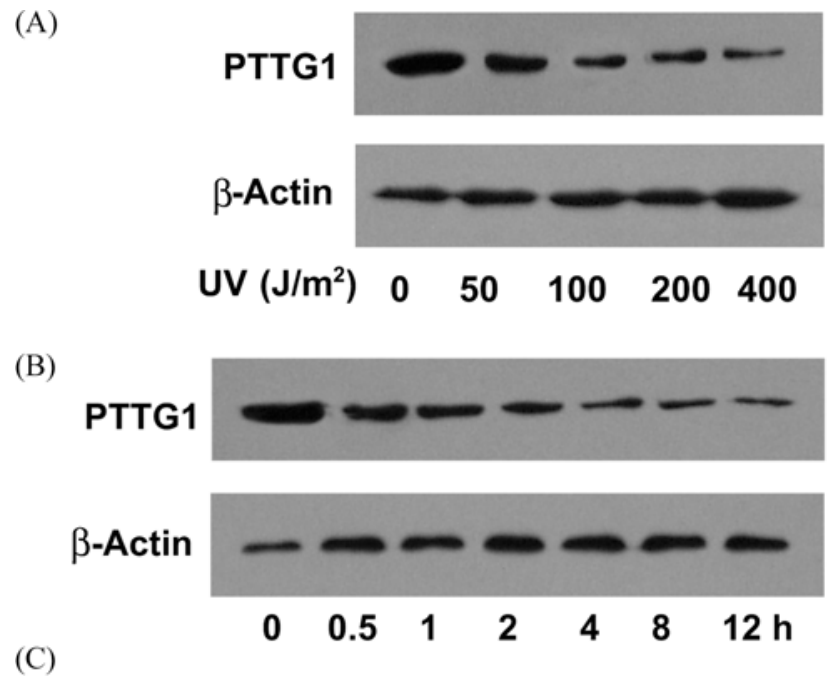

(C)

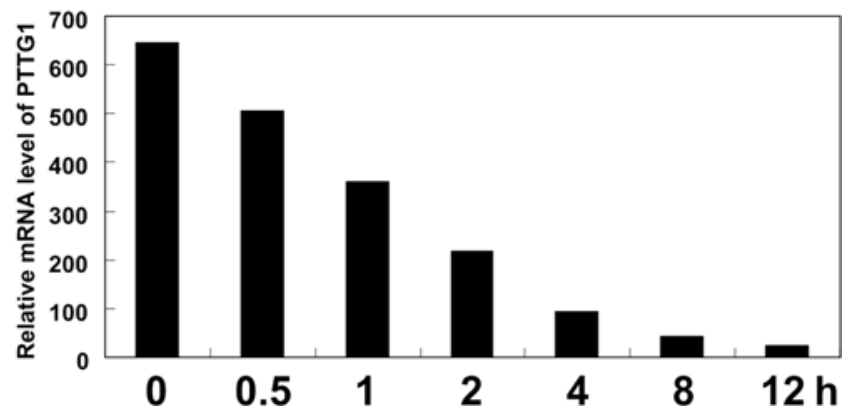

Fig. 3. The effect of UV irradiation on PTTG1 expression. (A) After UV irradiation of different doses, the cells were reincubated in DMEM media for $12 \mathrm{~h}$ and then harvested to examine with western blot. (B) and (C): Hela cells were exprosed to UV irradiation at the dose of $400 \mathrm{~J} / \mathrm{m}^{2}$, then reincubated in DMEM. They were harvested at the indicated times to determine the expression of PTTG1 protein and mRNA. (B) Western blot results. (C) Real-time quantitative PCR results.

whereas about $19 \%$ of other 2 cell types died through the apoptotic pathway (no apparent difference between these 2 cell lines). Together, these results suggest that overexpression of PTTG1 decreases UV-dependent apoptosis.

UV irradiation decreases PTTG1 mRNA and protein expression. As described above, PTTG1 protected HeLa cells against UV-induced apoptosis. Therefore, we reasoned that the expression of PTTG1 decreased after treatment with UV irradiation.

As shown in Fig. 3A, UV-C irradiation resulted in a reduced amount of PTTG1 protein in HeLa cells as detected by western blotting. The decrease in PTTG1 level was dose dependent and occurred rapidly, within $0.5 \mathrm{~h}$ after UV exposure (Fig. 3B).

As shown in Fig. 3C, UV-C irradiation resulted in reduced amount of PTTG1 mRNA as detected by real-time quantitive PCR and occurred rapidly, within $0.5 \mathrm{~h}$ after UV exposure. Therefore, UV-mediated depletion of PTTG1 depends on mRNA downregulation. 
(A)
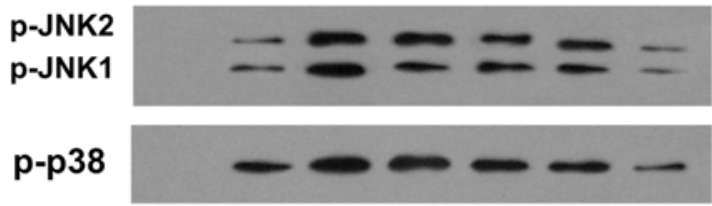

$\beta$-actin

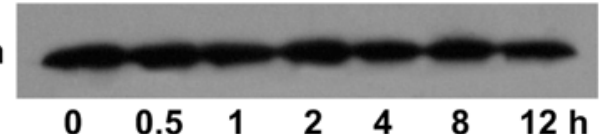

(B)
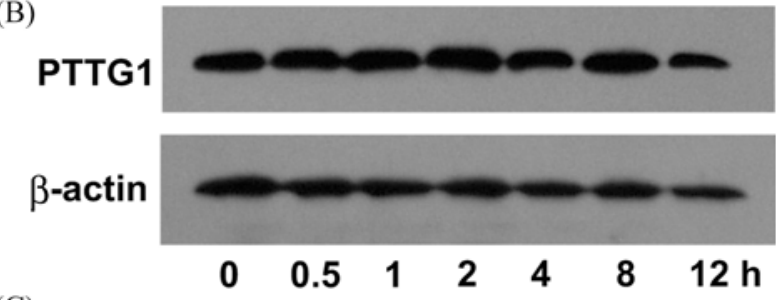

(C)

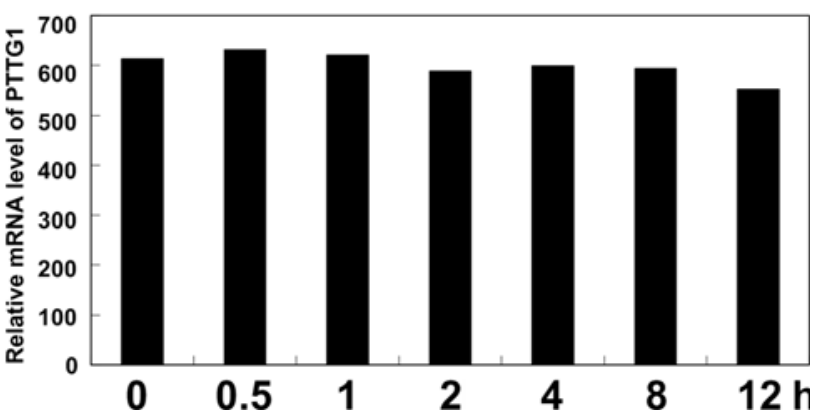

Fig. 4. The effects of c-Jun N-terminal kinase inhibitor (SP600125) on UV-induced decrease of PTTG1 protein and mRNA expression. (A) The effects of UV irradiation on activation of JNK and p38 pathway in Hela cells. They were UV-challenged $\left(400 \mathrm{~J} / \mathrm{m}^{2}\right)$ and reincubated in DMEM media. Then harvested at the indicated times. (B) The effects of c-Jun N-terminal kinase inhibitor (SP600125) on UV-induced decrease of PTTG1 protein expression. Hela cells were pretreated with SP600125 $(20 \mu \mathrm{M})$ for 0.5 to $1 \mathrm{~h}$, and then underwent UV irradiation at $400 \mathrm{~J} / \mathrm{m}^{2}$. They were reincubated in DMEM with $20 \mu \mathrm{M}$ SP600125. Cells were harvested at the indicated times to undergo western blot analysis.. SP600125 can inhibit the activations of p-JNK. (C) The effects of c-Jun N-terminal kinase inhibitor (SP600125) on UV-induced decrease of PTTG1 mRNA expression. Hela cells were pretreated with SP600125 $(20 \mu \mathrm{M})$ for 0.5 to $1 \mathrm{~h}$ and then underwent UV irradiation at $400 \mathrm{~J} / \mathrm{m}^{2}$. They were reincubated in DMEM with $20 \mu \mathrm{M}$ SP600125. Cells were harvested at the indicated times to undergo real-time PCR.

UV irradiation decreases PTTG1 mRNA and protein expression through the JNK pathway. Since UV irradiation decreased PTTG1 protein and mRNA expression, we wondered how UV irradiation downregulated PTTG1. UV irradiation activates several pathways, including the Jun Nterminal kinases (JNK) and p38 pathway, to induce apoptosis. Therefore, we tested whether UV irradiation activated these 2 pathways in the HeLa cell line. As shown in Fig. 4A, UV irradiation induced a rapid and sustained activation of both JNK and p38.

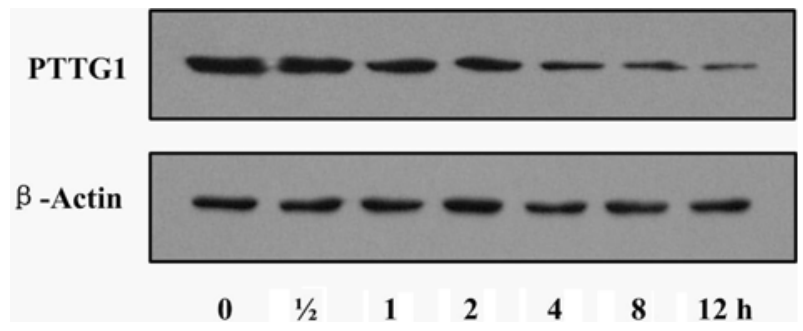

Fig. 5. The effects of p38 inhibitor (SB203580) on UV-induced decrease of PTTG1 protein expression. Hela cells were pretreated with SB203580 for 0.5 to $1 \mathrm{~h}$, then underwent UV irradiation at $400 \mathrm{~J} / \mathrm{m}^{2}$. They were reincubated in DMEM with $10 \mu \mathrm{M} \mathrm{SB} 203580$. Cells were harvested at the indicated times to undergo western blot analysis. SB203580 can inhibit the activations of p-p38.

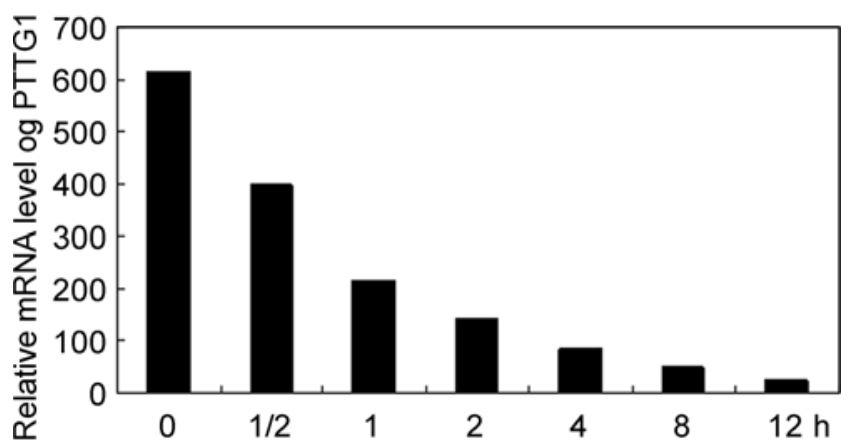

Fig. 6. The effects of p38 inhibitor (SB 203580) on UV-induced decrease of PTTG1 mRNA expression. Hela cells were pretreated with SB203580 for 0.5 to $1 \mathrm{~h}$ and then underwent UV irradiation at $400 \mathrm{~J} / \mathrm{m}^{2}$. They were reincubated in DMEM $10 \mu \mathrm{M}$ SB 203580. Cells were harvested at the indicated times to undergo real-time PCR.

To identify the mechanism involved in PTTG1 depletion, first we incubated UV-treated HeLa cells with the inhibitors of the JNK and p38 pathway (SP600125: c-Jun N-terminal kinase inhibitor; SB203580: p38 inhibitor). Treatment with SP600125 prevented the reduced amount of PTTG1 protein (Fig. 4B). However, SB 203580 was unable to prevent the reduction (Fig. 5).

Next, we examined the effect of SP600125 and SB 203580 on UV-mediated depletion of PTTG1 by real-time quantitive PCR. SP600125 (Fig. 4C) but not SB203580 (Fig. 6) prevented the reduction in the amount of PTTG1 mRNA. Therefore, UV irradiation decreases PTTG1 protein and mRNA expression through the JNK pathway.

\section{Discussion}

Our results showed that PTTG1 suppression increased and overexpression decreased UV-induced apoptosis. Furthermore, UV irradiation downregulated the PTTG1 mRNA and protein expression in a time- and dose-dependent manner. Finally, our results demonstrated that UV irradiation-induced suppression of PTTG1 was primarily through activation of the JNK signaling pathway. 


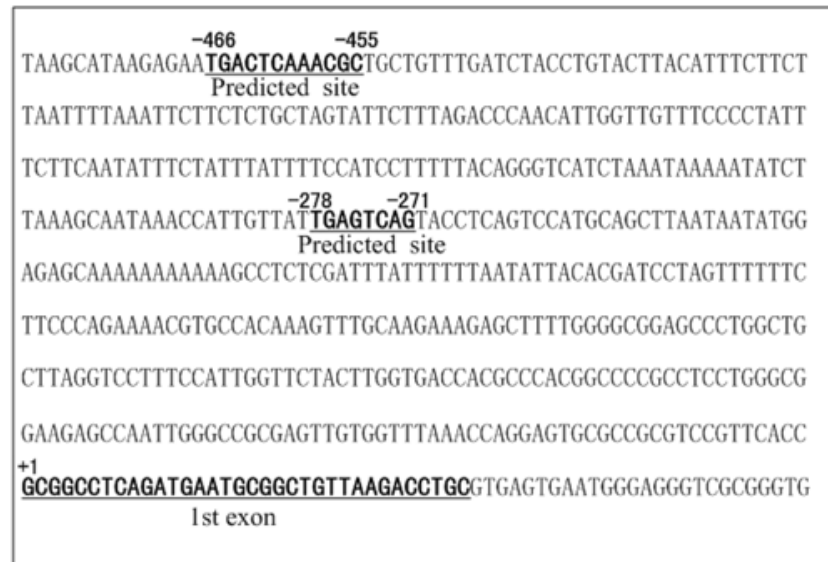

Fig. 7. Predicted c-Jun binding sites in the promoter region of PTTG1. It was performed with an online tool ${ }^{\mathrm{TM}}$ MAPPER (http:// mapper.chip.org/). There are two most possible binding sites of cJun in the promoter of PTTG1. The transcript start site is marked with " +1 " and the number of these two Predicted c-Jun binding sites are relative to The transcript start site.

In our experiments, knockdown of PTTG1 sensitized HeLa cells to the UV-induced apoptosis, and overexpression of PTTG1 decreased UV-dependent apoptosis. These results showed that the PTTG1 gene protected HeLa cells against UV damage-mediated apoptosis. Kho and colleagues showed that cells depleted of PTTG1 were more sensitive to 5-FU(5fluoro-2,4(1H,3H)pyrimidinedione)-induced apoptosis(Kho et al., 2004). As both UV irradiation and 5-FU can cause DNA damage, therefore our results, in conjunction with Kho's data showed that the PTTG1 gene protected cells against DNA damage-mediated apoptosis. Therefore, PTTG1 had an important anti-apoptotic role in DNA damage-induced apoptosis.

UV-induced apoptosis is a beneficial event that allows the host to eliminate cells containing DNA damage and thwart the accumulation of genetic mutations. As we described previously, PTTG1 is an anti-apoptotic gene in UV-induced apoptosis. So we wondered about the effect on PTTG1 expression of exposure to UV irradiation? Our further experiments showed that UV irradiation decreased PTTG1 protein and mRNA expression, which allows cells to undergo apoptosis when the DNA damage is too serious to repair. By eliminating cells with unrepairable DNA damage, the host may avoid accumulating mutations caused by UV irradiation and the ensuing development of cancers, especially skin cancer. Therefore, after exposure to UV irradiation, signaling mechanisms must downregulate the expression of PTTG1 to induce apoptosis.

Cells respond to UV irradiation exposure with strictly regulated inter- and intracellular signaling mechanisms. Mitogen-activated protein kinases (MAPK) play crucial roles in this response. In humans, at least 3 MAPK family proteins are involved in the UV-irradiation response: extracellular signal-regulated kinase (ERK), p38, and JNK. JNK activates $\mathrm{N}$-terminus of the transcription factor c-Jun and other Jun- family proteins through phosphorylation (Derijard et al., 1994; Kyriakis et al., 1994; Whitmarsh and Davis, 1996). In our experiment, decreased PTTG1 mRNA and protein expression on UV irradiation was mediated by the JNK pathway. Although the exact mechanism of the regulation of PTTG1 by the JNK pathway was still unclear, the results implied that on exposure to UV irradiation, JNK was activated to phosphorylate c-Jun and other Jun-family proteins, then the latter regulated PTTG1 expression through affecting its transcription (Fig. 7).

In conclusion, we reported that PTTG1 had an important anti-apoptotic role in UV-induced apoptosis and this role was mediated by JNK pathway. These results may provide important information for understanding the exact role and the potential mechanism of PTTG1 in UV-induced apoptosis.

\section{References}

Boelaert, K., McCabe, C. J., Tannahill, L. A., Gittoes, N. J., Holder, R. L., Watkinson, J. C., Bradwell, A. R., Sheppard, M. C. and Franklyn, J. A. (2003) Pituitary tumor transforming gene and fibroblast growth factor-2 expression: potential prognostic indicators in differentiated thyroid cancer. J. Clin. Endocrinol. Metab. 88, 2341-2347.

Clem, A. L., Hamid, T. and Kakar, S. S. (2003) Characterization of the role of Sp1 and NF-Y in differential regulation of PTTG/ securin expression in tumor cells. Gene 322, 113-121.

Derijard, B., Hibi, M., Wu, I. H., Barrett, T., Su, B., Deng, T., Karin, M. and Davis, R. J. (1994) JNK1: a protein kinase stimulated by UV light and Ha-Ras that binds and phosphorylates the c-Jun activation domain. Cell 76, 1025-1037.

Dominguez, A., Ramos-Morales, F., Romero, F., Rios, R. M., Dreyfus, F., Tortolero, M. and Pintor-Toro, J. A. (1998) Hpttg, a human homologue of rat pttg, is overexpressed in hematopoietic neoplasms. Evidence for a transcriptional activation function of hPTTG. Oncogene 17, 2187-2193.

Hamid, T., Malik, M. T. and Kakar, S. S. (2005) Ectopic expression of PTTG1/securin promotes tumorigenesis in human embryonic kidney cells. Mol. Cancer 4, 3 .

Heaney, A. P., Singson, R., McCabe, C. J., Nelson, V., Nakashima, M. and Melmed, S. (2000) Expression of pituitary-tumour transforming gene in colorectal tumours. Lancet 355, 716-719.

Hwang, H., Choi, S. Y. and Kim, T. Y. (2007) IL-4 suppresses UVBinduced apoptosis in skin. J. Biochem. Mol. Biol. 40, 36-43.

Jallepalli, P. V., Waizenegger, I. C., Bunz, F., Langer, S., Speicher, M. R., Peters, J. M., Kinzler, K. W., Vogelstein, B. and Lengauer, C. (2001) Securin is required for chromosomal stability in human cells. Cell 105, 445-457.

Kakar, S. S. and Jennes, L. (1999) Molecular cloning and characterization of the tumor transforming gene (TUTR1): a novel gene in human tumorigenesis. Cytogenet. Cell Genet. 84, 211-216.

Kho, P. S., Wang, Z., Zhuang, L., Li, Y., Chew, J. L., Ng, H. H., Liu, E. T. and Yu, Q. (2004) P53-regulated transcriptional program associated with genotoxic stress-induced apoptosis. J. Biol. Chem. 279, 21183-21192.

Kyriakis, J. M., Banerjee, P., Nikolakaki, E., Dai, T., Rubie, E. A., 
Ahmad, M. F., Avruch, J. and Woodgett, J. R. (1994) The stressactivated protein kinase subfamily of c-Jun kinases. Nature $\mathbf{3 6 9}$, 156-160.

McCabe, C. J., Khaira, J. S., Boelaert, L. K., Heaney, A. P., Tannahill, L. A., Hussain, S., Mitchell, R., Olliff, J., Sheppard, M. C., Franklyn, J. A. and Gittoes, N. J. (2003) Expression of pituitary tumour transforming gene (PTTG) and fibroblast growth factor-2 (FGF-2) in human pituitary adenomas: relationships to clinical tumour behaviour. Clin. Endocrinol. (Oxf) 58, 141-150.

Nakagawa, Y., Okada, S., Hatano, M., Ebara, M., Saisho, H. and Tokuhisa, T. (2002) Downregulation of bcl-xL is relevant to UVinduced apoptosis in fibroblasts. J. Biochem. Mol. Biol. 35, 452458.

Ogbagabriel, S., Fernando, M., Waldman, F. M., Bose, S. and Heaney, A. P. (2005) Securin is overexpressed in breast cancer. Mod. Pathol. 18, 985-990.

Pei, L. (2001) Identification of c-myc as a down-stream target for pituitary tumor-transforming gene. J. Biol. Chem. 276, 84848491.

Pei, L. and Melmed, S. (1997) Isolation and characterization of a pituitary tumor-transforming gene (PTTG). Mol. Endocrinol. 11, 433-441.

Ramos-Morales, F., Dominguez, A., Romero, F., Luna, R., Multon, M. C., Pintor-Toro, J. A. and Tortolero, M. (2000) Cell cycle regulated expression and phosphorylation of hpttg protooncogene product. Oncogene 19, 403-409.

Saez, C., Japon, M. A., Ramos-Morales, F., Romero, F., Segura, D. I., Tortolero, M. and Pintor-Toro, J. A. (1999) Hpttg is overexpressed in pituitary adenomas and other primary epithelial neoplasias. Oncogene 18, 5473-5476.

Saez, C., Martinez-Brocca,M. A., Castilla, C., Soto, A., Navarro, E., Tortolero, M., Pintor-Toro, J. A. and Japon, M. A. (2006) Prognostic Significance of hPTTG Immunohistochemical Expression in Differentiated Thyroid Cancer. J. Clin. Endocrinol. Metab. 91(4), 1404-1409.

Shibata, Y., Haruki, N., Kuwabara, Y., Nishiwaki, T., Kato, J., Shinoda, N., Sato, A., Kimura, M., Koyama, H., Toyama, T., Ishiguro, H., Kudo, J., Terashita, Y., Konishi, S. and Fujii, Y. (2002) Expression of PTTG (pituitary tumor transforming gene) in esophageal cancer. Jpn. J. Clin. Oncol. 32, 233-237.

Solbach, C., Roller, M., Fellbaum, C., Nicoletti, M. and Kaufmann, M. (2004) PTTG mRNA expression in primary breast cancer: a prognostic marker for lymph node invasion and tumor recurrence. Breast 13, 80-81.

Tfelt-Hansen, J., Yano, S., Bandyopadhyay, S., Carroll, R., Brown, E. M. and Chattopadhyay, N. (2004) Expression of pituitary tumor transforming gene (PTTG) and its binding protein in human astrocytes and astrocytoma cells: function and regulation of PTTG in U87 astrocytoma cells. Endocrinology 145, 4222-4231.

Wang, Z., Yu, R. and Melmed, S. (2001) Mice lacking pituitary tumor transforming gene show testicular and splenic hypoplasia, thymic hyperplasia, thrombocytopenia, aberrant cell cycle progression, and premature centromere division. Mol. Endocrinol. 15, 1870-1879.

Whitmarsh, A. J. and Davis, R. J. (1996) Transcription factor AP-1 regulation by mitogen-activated protein kinase signal transduction pathways. J. Mol. Med. 74, 589-607.

Yu, Q., La Rose, J., Zhang, H., Takemura, H., Kohn, K. W. and Pommier, Y. (2002) UCN-01 inhibits p53 up-regulation and abrogates gamma-radiation-induced G(2)-M checkpoint independently of $\mathrm{p} 53$ by targeting both of the checkpoint kinases, Chk2 and Chk1. Cancer Res. 62, 5743-5748.

Yu, R., Ren, S. G., Horwitz, G. A., Wang, Z. and Melmed, S. (2000) Pituitary tumor transforming gene (PTTG) regulates placental JEG-3 cell division and survival: evidence from live cell imaging. Mol. Endocrinol. 14, 1137-1146.

Zhang, X., Horwitz, G. A., Heaney, A. P., Nakashima, M., Prezant, T. R., Bronstein, M. D. and Melmed, S. (1999a) Pituitary tumor transforming gene (PTTG) expression in pituitary adenomas. $J$. Clin. Endocrinol. Metab. 84, 761-767.

Zhang, X., Horwitz, G. A., Prezant, T. R., Valentini, A., Nakashima, M., Bronstein, M. D. and Melmed, S. (1999b) Structure, expression, and function of human pituitary tumor-transforming gene (PTTG). Mol. Endocrinol. 13, 156-66.

Zou, H., McGarry, T. J., Bernal, T. and Kirschner, M. W. (1999) Identification of a vertebrate sister-chromatid separation inhibitor involved in transformation and tumorigenesis. Science 285, 41822. 